\title{
Effects of Neonatal Stress and Morphine on Kappa Opioid Receptor Signaling
}

\author{
Thuy N. Vien ${ }^{\text {a Christine A. Gleason }}{ }^{\text {a }}$ Sarah L. Hays ${ }^{\text {a }}$ Ronald J. McPherson ${ }^{a}$ \\ Charles Chavkin $^{\text {b Sandra E. Juul }}{ }^{\mathrm{a}}$
}

Departments of a Pediatrics and ${ }^{b}$ Pharmacology, University of Washington, Seattle, Wash., USA

\section{Key Words}

Kappa opioid receptor $\cdot$ Neonatal stress $\cdot$ Morphine $\cdot$

Prodynorphin

\begin{abstract}
Background: Critically ill neonates experience multiple stressors during hospitalization. Opioids are commonly prescribed to ameliorate their pain and stress. However, the enduring effects of stress and opioids are not understood. The kappa opioid system is important in the mediation of stress in adults, but little is known about its function in neonates. Objectives: To characterize kappa opioid receptor (KOR) distribution in the neonatal mouse brain and test whether neonatal exposure to morphine, stress, or both, change KOR signaling. Methods: Five groups of wild-type C57BL/6 or prodynorphin (Pdyn) knockout mice were tested: (1) untreated control (dam-reared, no handling), (2) saline-injected control, (3) morphine-injected control, (4) stressed with saline injections and (5) stressed with morphine injections. Mice were treated from postnatal day 5 to postnatal day 9 , after which their brains were immunolabeled with a phospho-specific KOR antibody (KOR-P), glial fibrillary acidic protein or glutamic acid decarboxylase. Results: There were no effects of saline or morphine injection on KOR-P immunoreactivity. Neonatal stress increased KOR-P labeling in wildtype brains ( $p<0.05$ ), but not in $\mathrm{Pdyn}^{-/}$animals. Mice exposed to stress and morphine showed region-specific increases in KOR-P immunoreactivity from 38 to 500\% ( $p<$ 0.05 to $p<0.001$ ), with marked gliosis. In stressed morphine-
\end{abstract}

treated $\mathrm{Pdyn}^{-/-}$animals, KOR-P immunoreactivity was absent, but gliosis increased compared to wild-type animals. Conclusions: Neonatal stress increases KOR activation via the dynorphin system. Neonatal stress plus morphine treatment further increased this response and also resulted in hippocampal gliosis. Enhanced gliosis noted in $\mathrm{Pdyn}^{-/}$animals suggests that the endogenous dynorphin may play a role in downregulating this inflammatory response.

Copyright $\odot 2009$ S. Karger AG, Basel

\section{Introduction}

Preterm infants in the neonatal intensive care unit are exposed to prolonged, inescapable stress. They are separated from their mothers, mechanically ventilated, handled repeatedly by strangers, gavage-fed, exposed to repeated painful procedures such as suctioning and blood drawing, and frequently undergo periods of oxidative stress such as hypoxia followed by hyperoxia (apnea). Morphine sulfate is often used to ameliorate neonatal stress in mechanically ventilated infants, but such treatment is controversial because of its questionable efficacy in lowering stress scores $[1,2]$, side effects $[3,4]$ and the lack of consensus on appropriate analgesic doses $[5,6]$. Morphine treatment may have additional effects in the newborn, affecting both immune function [7-10] and neurodevelopment [11]. In order to study the developmental consequences of neonatal stress and morphine therapy, we designed a rodent model that incorporates

\section{KARGER}

๑ 2009 S. Karger AG, Basel

Fax +4161306 1234 E-Mail karger@karger.ch www.karger.com www.karger.com/neo
Sandra E. Juul, MD

Division of Neonatology, Department of Pediatrics, University of Washington 1959 NE Pacific Street, HSB RR449, UW Box 356320

Seattle, WA 98195-6320 (USA)

Tel. +1 206616 1059, Fax +1 206543 8926, E-Mail sjuul@u.washington.edu 
repeated maternal separation, drug injection, oxidant stress, gavage feedings and incidental handling to mimic the human neonatal intensive care experience $[12,13]$. To target the period of brain development of interest (24-30 weeks of gestation in humans), mice were stressed from postnatal day 5 (P5) through P9 [14]. We previously found that these neonatal treatments produced enduring reductions in adult arousal and disrupted learning [12, 13]. These findings raise additional concerns about the lasting effects of neonatal stress and opiate use on neurodevelopment and cognitive function.

In adult animals, stressors such as trauma, social defeat and neuropathic pain can each trigger dynorphin release $[15,16]$. Dynorphin is an endogenous opioid peptide with a selective affinity for the kappa opioid receptor (KOR), a key mediator of the stress response [17, 18]. There are 3 types of opioid receptors, kappa, mu and delta, each with specific functions. Dynorphin peptides have limited analgesic activity compared with mu-selective opiates [19, 20]; KOR agonists, however, stimulate dysphoria and distress $[21,22]$. In rodents, KOR agonists reduce self-administration of cocaine and morphine [23, 24 ], and disrupt the cocaine-mediated elevation of dopamine and place-preference learning $[25,26]$. Inescapable stress enhances opiate and psychostimulant self-administration [27-29], but the effect can be prevented by a disruption of KOR signaling [17]. KOR antagonism also prevents the aversive behaviors triggered by repeated swim or foot-shock stress, thus supporting the hypothesis that KOR signaling mediates stress-induced dysphoria in adult mice $[19,23,24]$.

We hypothesized that the sedative effects of morphine would suppress the adverse effects of stress and reduce the activation of the dynorphin/KOR stress-response system in the brain [24]. The distribution and function of KORs in a neonatal mouse brain have not been previously studied. Therefore, the principal objectives of this study were to, firstly, characterize the anatomic and cellular distribution of functional KORs in the developing mouse brain, and then to determine whether neonatal stress with or without morphine treatment alters KOR signaling. We used wild-type C57BL/6 mice and prodynorphin knockout animals $\left(\mathrm{Pdyn}^{-/-}\right)$to determine the individual and interactive effects of stress and morphine, and to begin to determine the mechanisms of such responses. We found that the dynorphin/KOR system was functional in neonatal mice, but contrary to our prediction, morphine synergistically increased the adverse effects of stress exposure.

\section{Methods}

\section{Animals}

Adult wild-type C57BL/6 mice were purchased (Harlan, San Diego, Calif., USA) and housed under a 12-hour light-dark cycle with free access to food and water. Breeding was performed with 2 females per cage. Birth was recorded as P1, and the treatments were begun on P5. Litters were culled to a maximum of 7 mice per dam. Mortality and weight were monitored. All animal procedures were approved by the University of Washington's Animal Care and Use Committee. $\mathrm{Pdyn}^{-/-}$mice maintained on a C57BL/6 genetic background were generated and bred in the University of Washington's vivarium by methods previously described [17].

\section{Treatment Groups}

On P5, in order to examine the effects of neonatal stress and morphine, pups were distributed and earmarked to create 5 treatment groups: (1) untreated control (dam-reared, no handling), (2) saline-injected control, (3) morphine-injected control, (4) stressed with saline injections, and (5) stressed with morphine injections. Treatments were performed daily from P5 to P9. Experiments were done separately using cohorts of wild-type and $\mathrm{Pdyn}^{-/-}$animals.

Untreated control animals underwent minimal handling on P5 and at euthanasia on P9. Groups 2-5 received $10-\mu$ injections subcutaneously of either saline or morphine twice daily at 08:00 and 15:30 h. The morphine (Baxter, Deerfield, Ill., USA) dose was $2 \mathrm{mg} / \mathrm{kg}$ in a $10-\mu \mathrm{l}$ volume, based on the average daily litter weight. This dose produces circulating morphine levels that approximate the range measured in human preterm infants who are given intermittent or continuous intravenous morphine infusion [12]. Stressed pups (groups 4 and 5) were separated daily from the dam and isolated in individual containers in a veterinary warmer at $32^{\circ} \mathrm{C}$ from $08: 00$ to $16: 00 \mathrm{~h}$, thus experiencing both maternal and littermate separation. Pups were gavage-fed 50-150 $\mu$ l of rodent milk substitute 3 times daily (10:00, 12:00, 14:00 h) using a size $24 \times 1$ stainless steel animal feeding needle (Popper \& Sons, New Hyde Park, N.Y., USA). To simulate the oxidant stress of apnea, pups were exposed to hypoxia (100\% nitrogen for $1 \mathrm{~min}$ ) followed by hyperoxia (100\% oxygen for $5 \mathrm{~min}$ ) twice daily (08:00 and 15:30 h). Mice were then returned to the dam with their concurrent unstressed littermates each evening and allowed to nurse overnight ad libitum.

\section{Immunohistochemistry}

To determine the effects of stress and/or morphine on KOR activation, wild-type and $\mathrm{Pdyn}^{-/}$mice were euthanized on day 5 of treatment (P9). To establish the anatomic and cellular distribution of functional KORs in neonatal mice, we injected unstressed wild-type P9 animals with the selective KOR agonist trans-3, 4dichloro-N-methyl-N-[2-(1-pyrrolidinyl)cyclohexyl]benzeneace tamide (U50,488; Tocris, Ellisville, Mo., USA) at $10 \mathrm{mg} / \mathrm{kg}$ s.c. 30 min prior to death. Mice were anesthetized with isoflurane (Sigma, St. Louis, Mo., USA) and transcardially perfusion-fixed using fresh $4 \%$ paraformaldehyde in a neutral $0.1 \mathrm{M}$ PBS. The brains were quickly removed, immersion fixed for $3 \mathrm{~h}$, cryoprotected in $30 \%(\mathrm{w} / \mathrm{v})$ sucrose/PBS at $4{ }^{\circ} \mathrm{C}$ overnight and sectioned coronally $(50 \mu \mathrm{m})$ on a sliding microtome. Sections were first rinsed in a neutral Tris buffer $(3 \times 5 \mathrm{~min})$, and thereafter in Tris-buffered saline (TBS, $3 \times 5 \mathrm{~min}$ ), incubated in $2 \%$ avidin/TBS (30 min), 
rinsed $(3 \times 10 \mathrm{~min})$, incubated with $2 \%$ biotin/TBS $(30 \mathrm{~min})$, rinsed $(3 \times 10 \mathrm{~min})$ and incubated overnight at room temperature in diluted primary antibody (antibody diluent: TBS/0.1\% Triton $/ 10 \%$ NGS). The primary antibodies were a phospho-selective rabbit anti-KOR (KOR-P, 1:200) [17, 30, 33], a mouse anti-glutamic acid decarboxylase (GAD67, 1:200; Chemicon, Temecula, Calif., USA) or a mouse anti-glial fibrillary acidic protein (GFAP, 1:200; Sigma). Sections were again rinsed $(6 \times 10 \mathrm{~min})$, incubated ( $2 \mathrm{~h}$ at room temperature) with biotinylated anti-rabbit IgG (1:300; Vector, Burlingame, Calif., USA) and anti-mouse Alexa Fluor 555 (1:500; Molecular Probes, Eugene, Oreg., USA), rinsed $(6 \times 10 \mathrm{~min})$, incubated ( 2 hours at room temperature) with avidin D fluorescein (1:300; Vector) and anti-mouse Alexa Fluor 555 (1:500), rinsed $(2 \times 5 \mathrm{~min})$, air-dried, coverslipped with Vectashield (Vector) and sealed. Antibody specificity for the KOR-P antibody was verified as described previously [17, 30, 33, 34]. Control sections included incubation with no primary antibody. No staining was detected above background in the absence of a primary antibody. Alternate coronal sections from P9 wild-type control animals were stained with cresyl violet to examine brain morphology.

Immunofluorescence was photographed with a Leica SL confocal microscope (Nusloch, Germany) located at the W.M. Keck Imaging Facility at the University of Washington. A coronal slice of the anterior brain containing the cortex and nucleus accumbens was sequentially photographed for KOR-P immunoreactivity and the resulting images were assembled into a spatial representation of this region (fig. 1). A more posterior coronal slice was taken of the same brain in which the hippocampus and hypothalamus were included. Images of KOR-P immunoreactivity were taken in the same manner (fig. 2).

To determine the effects of neonatal treatment, blinded images taken from multiple brain regions of interest were saved for the quantification of differences in immunoreactivity. Images were captured using constant settings for brightness and exposure time across all study groups. Pixel intensity was measured with MetaMorph (Downingtown, Pa., USA) and quantification was based on pixel values of KOR-P or GFAP immunoreactivity.

\section{Statistical Analysis}

Univariate ANOVA was performed using SPSS software (SPSS, Chicago, Ill., USA). Both the main effects and interactions were evident and justified all subsequent post hoc comparisons. Data were expressed as means with standard error (SEM) and/or as proportional increases compared to the control. Two-tailed Dunnett's tests were used to correct for multiple comparisons using untreated animals as the control group. An alpha of $\mathrm{p} \leq 0.05$ was used.

\section{Results}

\section{KOR-P Expression and Distribution in Unstressed}

Neonatal Mice

To investigate the distribution of functional KOR in an unstressed neonatal mouse brain, we injected $\mathrm{P} 9$ animals with U50,488 and measured KOR-P. We examined 9 regions in these brains that may be affected by morphine treatment or stress exposure. These were the parietal cortex, hippocampus (CA1, CA3, molecular layer), dentate gyrus, nucleus accumbens (core and shell), hypothalamus and cerebellum. We observed that KOR-P was present in all of these regions, with variable distribution throughout the wild-type P9 brain (fig. 1, 2).

In the anterior sections, KOR-P immunoreactivity was most intense in the sensory and motor cortex, striatum and the shell and core of the nucleus accumbens (fig. 1). In other regions, KOR-P was distributed in a widely diffuse manner. In order to characterize the cellular distribution of KOR-P, sections were double-labeled with KOR-P and GFAP or GAD67, and examined at a higher magnification. In the parietal cortex and nucleus accumbens, KOR-P labeling was not associated with astrocytes but did co-localize with GAD67-positive cells, most notably in the nucleus accumbens (fig. $3 a-c)$. In the cortex, the majority of KOR-P labeling did not co-localize with either GFAP or GAD67. Pdyn ${ }^{-/}$mice did not exhibit KOR-P immunoreactivity unless injected with U50,488 (fig. 3d-g), confirming the specificity of the KOR-P antibody.

In the more posterior sections of the brain, KOR-P immunoreactivity was observed in the parietal cortex, hippocampus, dentate gyrus, basal ganglia and hypothalamus (fig. 2). KOR-P immunoreactivity was less intense in the thalamus and sensory cortex. In the hippocampus and the white matter of the cerebellum, KOR-P co-localized with GFAP-positive cells. In other regions, KOR-P co-localized with GAD67 (fig. 3).

KOR-P immunoreactivity in unstressed wild-type P9 mice without U50,488 stimulation was present but less intense, particularly in the cortex, striatum, nucleus accumbens, basal ganglia and hypothalamus. The background distribution of functional KOR-mice not treated with U50,488 was more widely diffuse.

\section{Effects of Morphine Alone}

To quantify the effects of 5 days of exposure to morphine, we compared the pixel intensities of KOR-P immunoreactivity between treated and untreated neonatal animals. Table 1 shows the effects of morphine treatment compared to untreated controls on KOR-P immunoreactivity; a statistical analysis of the normalized intensity data is presented. In the regions examined, we observed a trend toward increased immunoreactivity due to the mild stress from just handling and saline injections, with an increase of $8 \%$ in KOR-P immunoreactivity in salineinjected animals and a 16\% increase in morphine-injected animals over untreated mice. While these changes did 
Fig. 1. Distribution of activated kappa opioid receptors in a wild-type P9 anterior brain. Immunofluorescent phospho-specific labeling of KOR-P in an anterior brain 30 min after stimulation with U50,488. a Individual regions were imaged $(400 \times)$ and assembled into a spatial representation of the anterior brain. Alternate sections were stained with cresyl violet, and fluorescent images from the same brain region were overlayed. Green fluorescence indicates the location of activated KOR. Brain regions are identified in the contralateral hemisphere: $\mathrm{Cg}=$ cingulate cortex; $\mathrm{M}=$ motor cortex $\mathrm{CPu}=$ caudate putamen (striatum); AcbC = nucleus accumbens core; AcbSh = nucleus accumbens shell. b Enlargement of an individual micrograph showing specificity of KOR-P labeling to cell bodies and processes in the motor cortex. Sections with the primary antibody deleted showed no KOR-P immunoreactivity.

Fig. 2. Distribution of activated kappa opioid receptors in a wild-type P9 posterior brain. Phospho-specific labeling of KOR-P in a posterior brain $30 \mathrm{~min}$ after stimulation with U50,488. a Spatial representation of a coronal slice of brain containing the hippocampus and hypothalamus. Photomicrograph magnification $400 \times$. Brain regions are identified in the contralateral hemisphere: $\mathrm{M}=$ motor cortex; $\mathrm{DG}=$ dentate gyrus; CA1 field = CA1 of hippocampus; CA3 field = CA3 of hippocampus; $\mathrm{CPu}=$ caudate putamen (striatum); $\mathrm{DM}=$ dorsal medial nuclei of the hypothalamus. b Enlargement of an individual micrograph showing specificity of KOR-P labeling to cell bodies and processes in the motor cortex. Sections with the primary antibody deleted showed no KOR-P immunoreactivity.
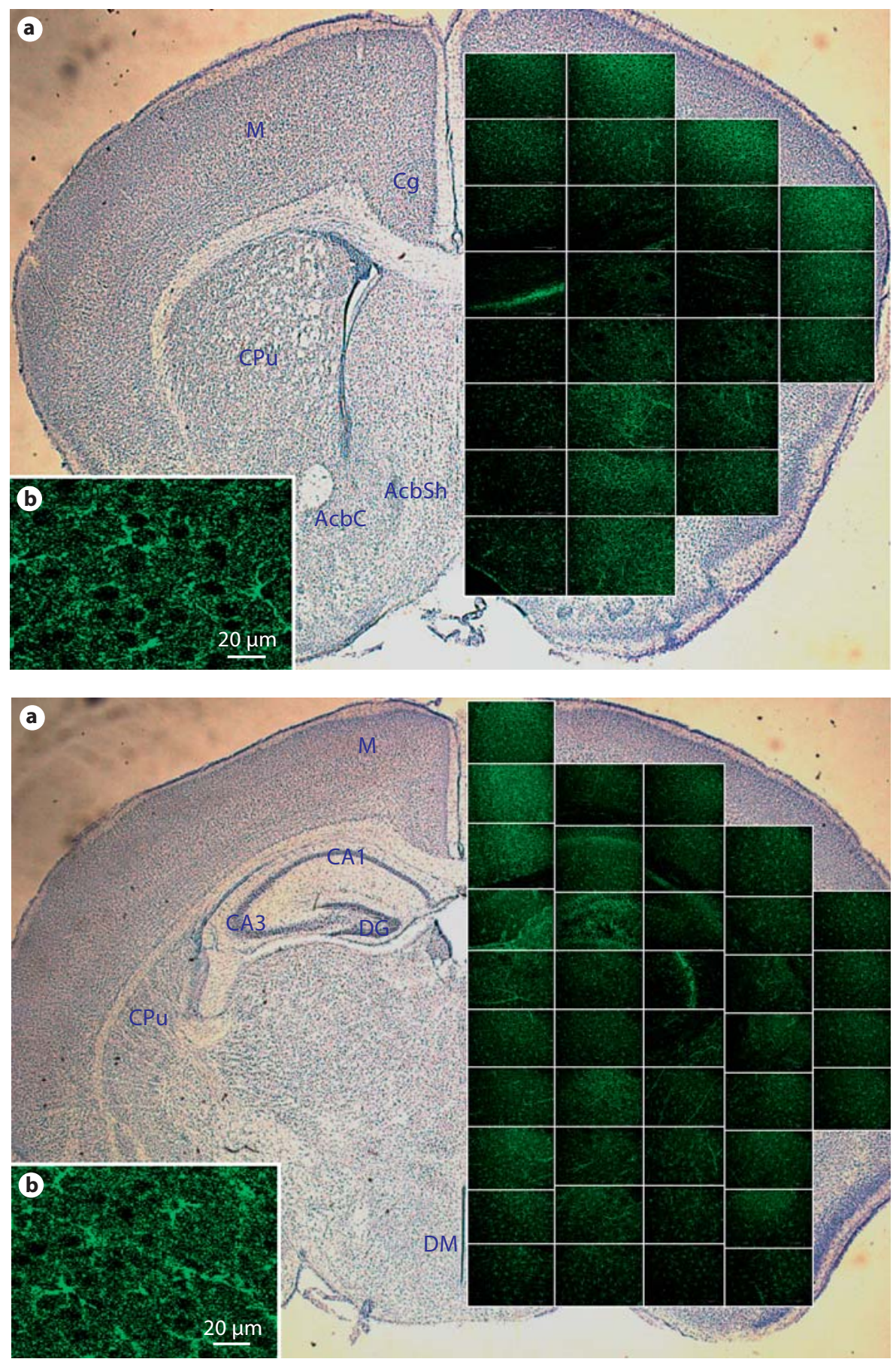

not reach statistical significance $(\mathrm{p}=0.06)$, they do suggest that the mild stress caused by handling and injections might increase KOR-P immunoreactivity. Morphine alone did not robustly activate the dynorphin/KOR system or produce significant changes in brain morphology.

\section{Effects of Neonatal Stress Alone}

Severe stress significantly increased KOR-P immunoreactivity in specific brain regions when compared to the control animals (table 1). Multiple areas within the hippocampus were strongly affected, with the CA3 layer (95\% increase), molecular layer (63\% increase), CA1 layer 

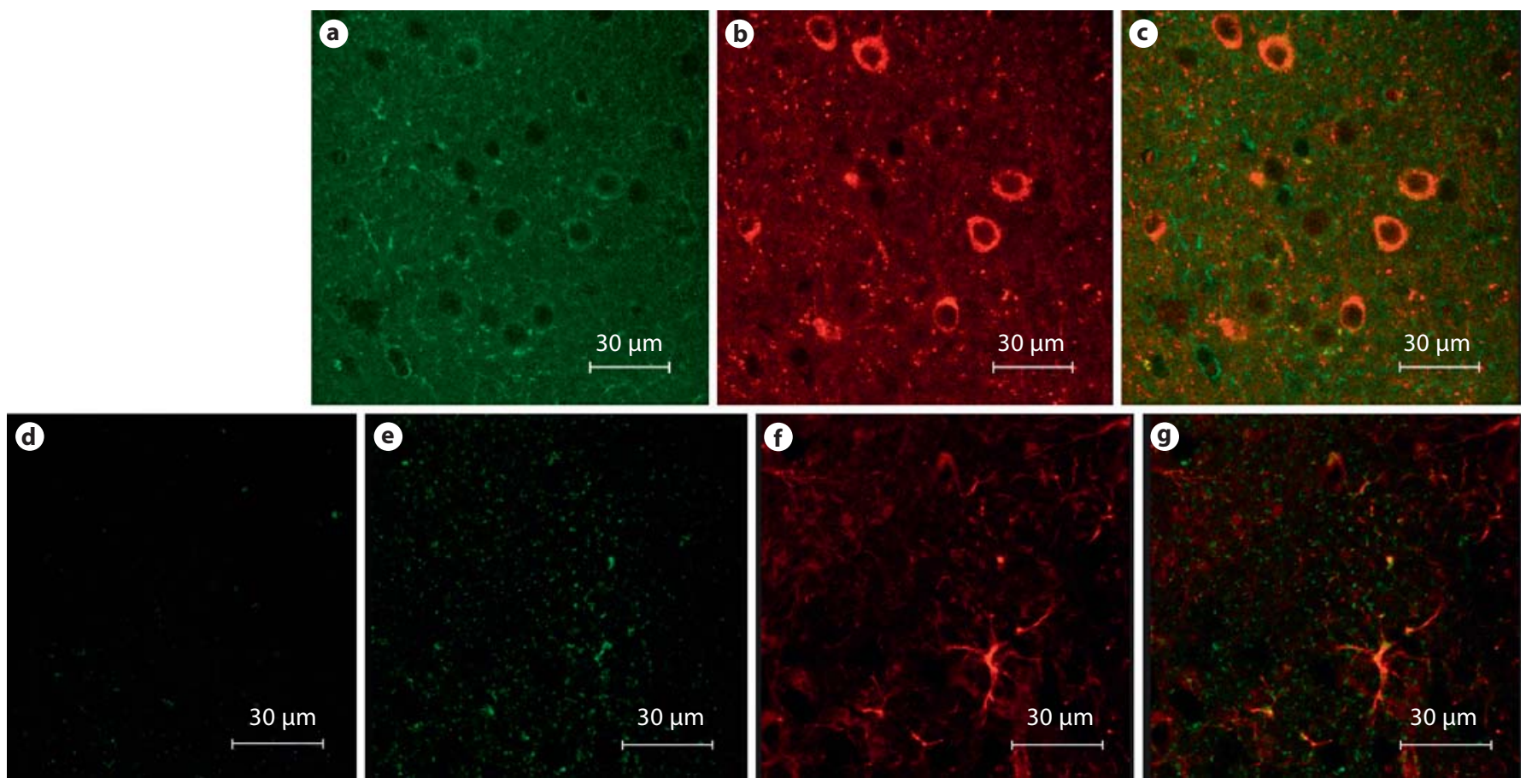

Fig. 3. KOR-P co-localizes to GAD67 ${ }^{+}$cells while $\mathrm{Pdyn}^{-/-}$mice do not exhibit KOR-P immunoreactivity unless injected with U50,488. a KOR-P immunoreactivity in the nucleus accumbens. b GAD67 immunoreactivity in the same region. c KOR-P co-localization with GAD67+ cells. $\mathrm{Pdyn}^{-1-}$ mice exhibited no KOR-P immunoreactivity above the background (d) while those injected with U50,488 showed KOR-P immunoreactivity (e), suggesting that kappa opioid receptors remain functional in $\mathrm{Pdyn}^{-/-}$animals. f GFAP immunoreactivity for the $\mathrm{Pdyn}^{-/-}$animal injected with U50,488. g Merged image of GFAP and KOR-P co-localization.

Table 1. Effects of neonatal morphine and/or stress on the mean ( \pm SEM; $n=4)$ KOR-P immunoreactivity pixel intensity (arbitrary units) in areas of the wild-type P9 mouse brain

\begin{tabular}{|c|c|c|c|c|c|}
\hline \multirow[t]{2}{*}{ Location } & \multicolumn{3}{|c|}{ Dam reared } & \multicolumn{2}{|l|}{ Stressed } \\
\hline & untreated & saline & morphine & saline & morphine \\
\hline Parietal cortex & $2.8 \pm 0.1$ & $3.0 \pm 0.1$ & $3.7 \pm 0.5$ & $2.9 \pm 0.0$ & $5.7 \pm 0.6^{* * *}$ \\
\hline Hippocampus, CA1 & $2.8 \pm 0.1$ & $2.9 \pm 0.0$ & $3.0 \pm 0.1$ & $3.8 \pm 0.4^{*}$ & $3.9 \pm 0.1^{* *}$ \\
\hline Hippocampus, CA3 & $3.0 \pm 0.2$ & $3.3 \pm 0.2$ & $3.4 \pm 0.2$ & $5.9 \pm 0.8^{* * *}$ & $4.3 \pm 0.4$ \\
\hline Hippocampus, DG & $3.0 \pm 0.2$ & $3.3 \pm 0.0$ & $3.5 \pm 0.1$ & $4.3 \pm 0.5^{*}$ & $4.7 \pm 0.4^{* *}$ \\
\hline Hippocampus, ML & $2.9 \pm 0.2$ & $3.0 \pm 0.1$ & $3.2 \pm 0.2$ & $4.7 \pm 0.8$ & $6.5 \pm 0.9^{* *}$ \\
\hline Nuclens accumbens, core & $3.0 \pm 0.2$ & $3.1 \pm 0.1$ & $3.5 \pm 0.2$ & $4.3 \pm 0.3^{* *}$ & $4.7 \pm 0.2^{* * *}$ \\
\hline Nuclens accumbens, shell & $3.1 \pm 0.2$ & $3.0 \pm 0.1$ & $3.4 \pm 0.1$ & $3.6 \pm 0.3$ & $18.8 \pm 2.9^{* * *}$ \\
\hline Hypothalamus & $2.8 \pm 0.1$ & $2.9 \pm 0.0$ & $3.4 \pm 0.1$ & $3.4 \pm 0.1$ & $5.2 \pm 0.3^{* * *}$ \\
\hline Cerebellum & $4.6 \pm 0.2$ & $6.3 \pm 0.3$ & $5.3 \pm 0.4$ & $10.7 \pm 2.1^{*}$ & $12.1 \pm 2.2^{* *}$ \\
\hline
\end{tabular}

ANOVA $\left(\operatorname{drug}_{3} \times\right.$ stress $\left._{2}\right)$ found significant treatment effects and/or interactions for gain-matched pixel intensity data at each separate location listed. Subsequent post hoc comparisons were made using Dunnett's test to compare each group to the untreated control group ( $n=4 /$ group).

Significant differences are shown as ${ }^{*} \mathrm{p}<0.05,{ }^{* *} \mathrm{p}<0.01$, or ${ }^{* *} \mathrm{p}<0.001$. DG = Dentate gyrus; $\mathrm{ML}=\mathrm{mo}-$ lecular layer. 

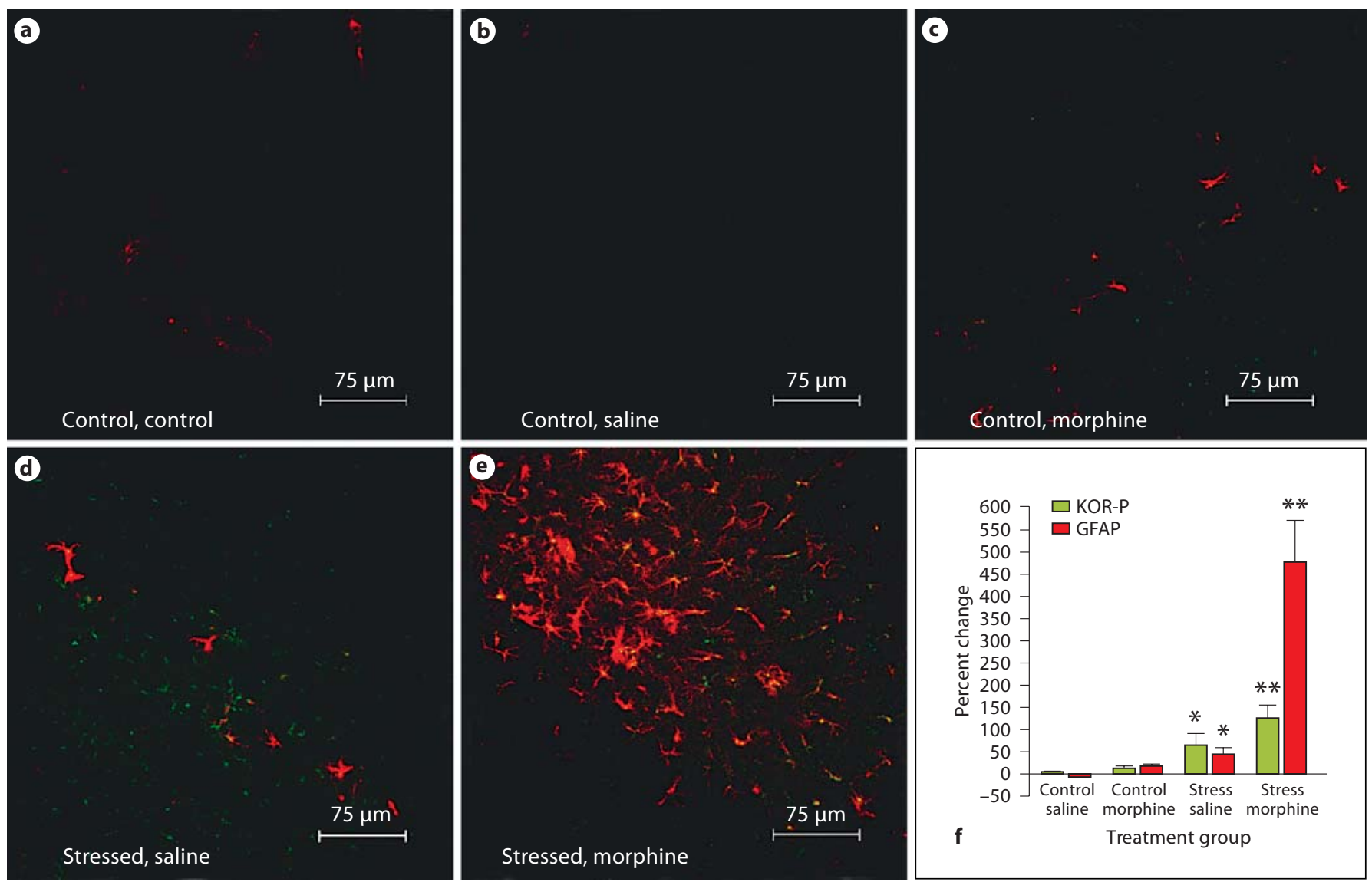

Fig. 4. KOR-P and GFAP immunoreactivity increases in the molecular layer of the hippocampus in wild-type neonatal mice exposed to stress and treated with morphine. The distribution of GFAP immunoreactivity (red) and activated KOR (green) is compared in the molecular layer of the hippocampus from 5 treatment groups: untreated control (a), saline-injected control (b), morphine-injected control (c), stressed with saline injections (d), and stressed with morphine injections (e). Photomicrograph magnification $400 \times$. $\mathbf{f}$ Percent change in pixel intensity for both GFAP and KOR-P in the molecular layer of the wild-type mouse hippocampus. Red bars represent the percent change in GFAP immunoreactivity, and green bars represent the percent change in KOR$\mathrm{P}$ immunoreactivity in the treatment groups compared to the untreated controls. ${ }^{*} \mathrm{p}<0.05 ;{ }^{* *} \mathrm{p}<0.01$.
(34\% increase) and dentate gyrus (41\% increase) all showing marked increases in immunofluorescence. The nucleus accumbens core ( $43 \%$ increase) and cerebellum (134\% increase) also showed increased KOR activation. In contrast, KOR-P immunoreactivity in the cortex, nucleus accumbens shell and hypothalamus was not different in the stressed animals compared to the untreated control animals. Overall, KOR-P immunoreactivity increased by an average of $51 \%$ compared to the control animals and $38 \%$ over animals that experienced the mild stress of handling and injections.

Neonatal stress, by itself, increased GFAP immunoreactivity in the hippocampus as well. In the molecular layer of the hippocampus, a $43 \%$ increase in GFAP pixel in- tensity was observed in comparison to the control animals (fig. 4). KOR-P co-localized with GFAP in the molecular layer.

\section{Effects of Neonatal Stress with Morphine}

Animals exposed to both neonatal stress and morphine showed the greatest changes in KOR-P immunoreactivity, indicating an interaction between stress and morphine. KOR-P immunoreactivity in the molecular layer of the hippocampus was highly affected $(125 \%$ increase), which was more than the CA1 region (38\% increase) or dentate gyrus (55\% increase). Other substantial differences in KOR-P immunoreactivity for stressplus-morphine-treated mice (compared to control) were 

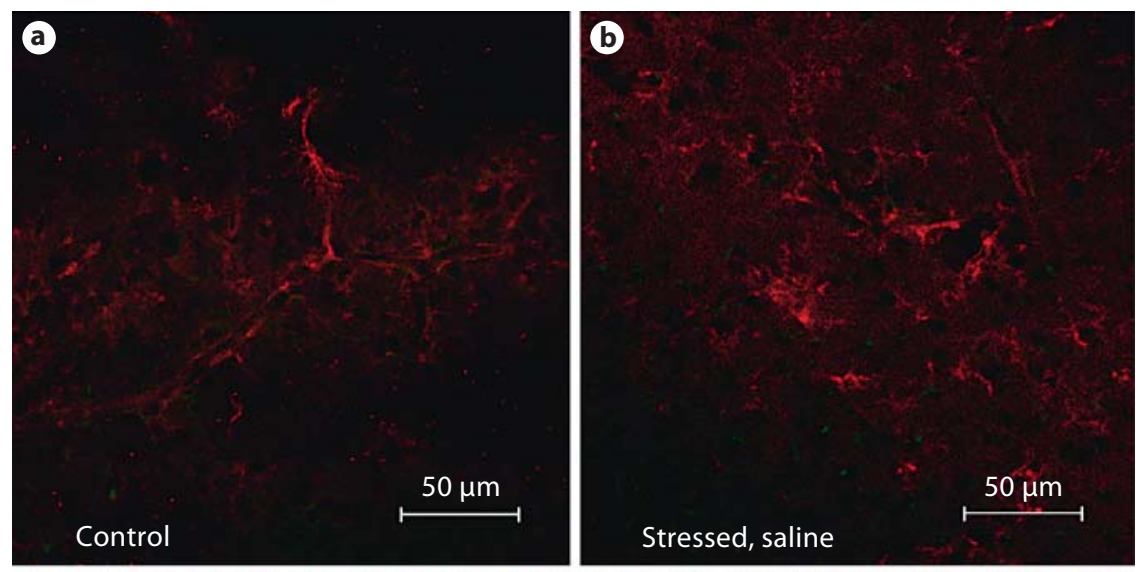

Fig. 5. KOR-P and GFAP immunoreactivity increased in the hippocampus in P9 $\mathrm{Pdyn}^{-/-}$ neonatal mice exposed to stress and treated with morphine. The distribution of GFAP immunoreactivity (red) and activated KOR (green) is compared in the molecular layer of the hippocampus from untreated control (a), stressed with saline injections (b), and stressed with morphine injections (c). d GFAP immunoreactivity (red) and activated KOR immunoreactivity (green) in the CA3 field of the hippocampus in stressed, morphine-injected mice. Photomicrograph magnification $640 \times$.
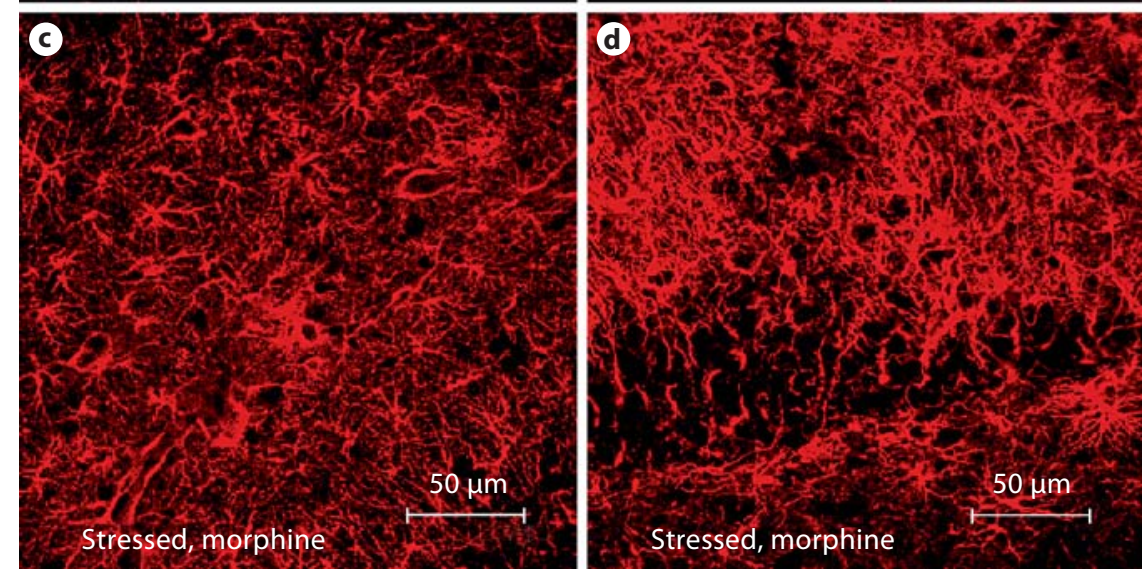

observed in the cortex (100\% increase), the nucleus accumbens core (58\% increase) and shell (500\% increase), the hypothalamus ( $85 \%$ increase), and the cerebellum (165\% increase). In contrast to the wild-type animals, $\mathrm{Pdyn}^{-/-}$animals exhibited no KOR-P immunoreactivity under any treatment condition, including exposure to severe stress and morphine treatment. Presence of functional KOR in these animals was confirmed by stimulation with U50,488 (fig. 3). This suggests that the effects of neonatal stress and morphine on KOR activation are mediated by the stress-induced release of endogenous dynorphin.

Neonatal stress combined with morphine treatment resulted in an even greater increase in GFAP immunoreactivity than stress alone. In wild-type animals, the molecular layer of the hippocampus showed a $474 \%$ increase in GFAP immunoreactivity. KOR-P co-localized predominantly to GFAP-positive cells (fig. 4). In $\mathrm{Pdyn}^{-1-}$ animals, this gliotic response was even more exuberant. Unlike the gliosis in wild-type mice that was confined primarily to the molecular layer of the hippocampus,
$\mathrm{Pdyn}^{-/-}$mice exposed to stress plus morphine treatment demonstrated extensive GFAP immunoreactivity throughout the hippocampus (fig. 5), widely crossing into the granule and pyramidal cell layers.

\section{Discussion}

We have characterized the anatomic and cellular distribution of functional KORs in the developing mouse brain and shown that neonatal stress increases KOR signaling, with stress plus morphine having an even more robust effect, as evidenced by increased immunoreactivity with a phospho-specific antibody. We have also shown a regional gliotic response to neonatal stress, which is also more pronounced when neonatal stress is treated with morphine.

In wild-type animals, the kappa opioid system was active in the baseline state, but immunoreactivity increased when stimulated with U50,488. In contrast, in $\mathrm{Pdyn}^{-/-}$ animals, KOR-P immunoreactivity was only evident af- 
ter stimulation with U50,488, indicating that the endogenous dynorphin is required for receptor activation, but does not affect receptor distribution. In adult rodents, KORs are present in both neurons and glia [27-29], as indicated by co-localization with GAD67-positive neurons and GFAP-positive astrocytes. We showed similar patterns of co-localization during the neonatal period. A functional kappa opioid system has not previously been reported in a neonatal brain. In adult rat and mouse studies, the presence of functional KORs has been well established in the cortex, nucleus accumbens, midbrain regions and in the cerebellar cortex [30]. We have established a comparable presence of KORs in neonates.

In this study we tested whether stress, morphine or stress plus morphine affected KOR signaling. Five days of neonatal morphine treatment did not significantly alter KOR-P distribution or intensity. In contrast, 5 days of neonatal stress increased KOR-P immunoreactivity in the hippocampus, the nucleus accumbens core and the cerebellum. Stress plus morphine further increased KOR$\mathrm{P}$ labeling in wild-type, but not in $\mathrm{Pdyn}^{-/-}$mice, suggesting that dynorphin is required for this stress-related increase in KOR-P signaling. Stress plus morphine treatment also induced gliosis in the molecular layer of the hippocampus of wild-type mice, with KOR-P co-localizing with GFAP. Gliosis is a hallmark of inflammation and may indicate subsequent damage. This stress-induced hippocampal gliosis is a novel finding that may indicate a vulnerability of the hippocampus to early stress-induced injury and explain corresponding learning impairments in adults. In contrast to the effect of stress and morphine on activation of KOR (dynorphin had a stimulatory effect), gliosis was exacerbated in $\mathrm{Pdyn}^{-/}$mice, suggesting that dynorphin negatively regulates this response.

We initiated this study to investigate the role of the kappa opioid system in mediating the long-term behavioral consequences of neonatal stress exposure and early morphine treatment. An estimated 52,600 preterm or low-weight infants are born each year in the United States [31], with the majority remaining hospitalized for weeks or even months in neonatal intensive care units. Even though caution is advised with the use of morphine in preterm infants, this drug is still commonly used as a sedative in these infants despite evidence showing that morphine analgesia is not always effective [32], can trigger hypotension [3, 4], and may increase the risk of a poor outcome $[2,3]$. The rationale to use morphine to alleviate stress is weakened by the observations that stress scores from infants given morphine are contradictory $[1,33]$ and that adrenal stress response is attenuated by morphine in adult rodents, but not in neonates. There are concerns that opioids might interact adversely with stressors or perturb the natural sequence of neurogenesis.

We have shown that neonatal stress in conjunction with morphine synergistically activates the kappa opioid system, as demonstrated by the increased KOR-P immunoreactivity. Increases in KOR-P pixel intensity have previously been shown to reflect an increase in GRK3phosphorylated KOR concentration [34]. However, the increased labeling could also reflect an increase in the activity of pre-existing kappa receptor populations. The long-term consequences of altered neonatal KOR signaling are unknown and may be important for the enduring behavioral changes we have previously reported [12, 13]. Given that KOR signaling regulates cocaine self-administration in adults, we can hypothesize that the up-regulation of KOR resulting from stress and morphine treatment may alter adult drug-seeking behavior. Dynorphin dysfunction has been implicated in drug dependence and schizophrenia [34, 35], and dynorphin expression in limbic nuclei is directly modulated by pain and stress [36]. The gliosis associated with neonatal stress and morphine treatment appeared to be down-regulated by dynorphin, since the knockout animals showed a more exuberant gliotic response. The current report has established that the kappa opioid system plays an important role in the neonatal response to stress and morphine and may also mediate the long-term effects of these neonatal treatments on adult behavior. Future experiments are warranted to define the lasting effects of neonatal treatments on KORmediated adult drug-seeking or reward-conditioned learning behaviors.

\section{Acknowledgements}

The authors would like to thank Greg Martin of the W.M. Keck Imaging Facility at the University of Washington, and Paul G. Decaen for critical reading of the manuscript. This study was financially supported by grants from Children's Hospital and Medical Center, Seattle Basic Science Steering Committee and USPHS grants DA22573 and DA16898 from the National Institute on Drug Abuse. 


\section{References}

1 Quinn MW, Wild J, Dean HG, Hartley R, Rushforth JA, Puntis JW, Levene MI: Randomised double-blind controlled trial of effect of morphine on catecholamine concentrations in ventilated pre-term babies. Lancet 1993;342:324-327.

-2 Simons SH, van Dijk M, van Lingen RA, Roofthooft D, Duivenvoorden HJ, Jongeneel N, Bunkers C, Smink E, Anand KJ, van den Anker JN, Tibboel D: Routine morphine infusion in preterm newborns who received ventilatory support: a randomized controlled trial. JAMA 2003;290:2419-2427.

-3 Anand KJ, Hall RW, Desai N, Shephard B, Bergqvist LL, Young TE, Boyle EM, Carbajal R, Bhutani VK, Moore MB, Kronsberg SS, Barton BA: Effects of morphine analgesia in ventilated preterm neonates: primary outcomes from the NEOPAIN randomised trial. Lancet 2004;363:1673-1682.

-4 Hall RW, Kronsberg SS, Barton BA, Kaiser JR, Anand KJ: Morphine, hypotension, and adverse outcomes among preterm neonates: who's to blame? Secondary results from the NEOPAIN trial. Pediatrics 2005; 115:13511359.

5 Ambalavanan N, Carlo WA: Analgesia for ventilated neonates: where do we stand? J Pediatr 1999;135:403-405.

-6 Kennedy KA, Tyson JE: Narcotic analgesia for ventilated newborns: are placebo-controlled trials ethical and necessary? J Pediatr 1999;134:127-129.

7 Yossuck P, Nightengale BJ, Fortney JE, Gibson LF: Effect of morphine sulfate on neonatal neutrophil chemotaxis. Clin J Pain 2008; 24:76-82.

8 Guo LH, Schluesener HJ: The innate immunity of the central nervous system in chronic pain: the role of toll-like receptors. Cell Mol Life Sci 2007;64:1128-1136.

\9 Jamali A, Bamdad T, Soleimanjahi H, Pakdel FG, Arefian E: Acute morphine administration reduces white blood cells' capability to induce innate resistance against hsv-1 infection in balb/c mice. Neuroimmunomodulation 2007;14:16-23.

-10 Saurer TB, Ijames SG, Carrigan KA, Lysle DT: Neuroimmune mechanisms of opioidmediated conditioned immunomodulation. Brain Behav Immun 2008;22:89-97.

11 Anand KJ, Scalzo FM: Can adverse neonatal experiences alter brain development and subsequent behavior? Biol Neonate 2000;77: 69-82.

-12 McPherson RJ, Gleason C, Mascher-Denen M, Chan M, Kellert B, Juul SE: A new model of neonatal stress which produces lasting neurobehavioral effects in adult rats. Neonatology 2007;92:33-41.
13 Boasen JF, McPherson RJ, Hays SL, Juul SE, Gleason CA: Effect of neonatal stress and/or morphine treatment on behavior of adult mice. Neonatology 2008;95:230-239.

14 Clancy B, Kersh B, Hyde J, Darlington RB, Anand KJ, Finlay BL: Web-based method for translating neurodevelopment from laboratory species to humans. Neuroinformatics 2007;5:79-94.

-15 Przewlocki R, Lason W, Hollt V, Silberring J, Herz A: The influence of chronic stress on multiple opioid peptide systems in the rat: pronounced effects upon dynorphin in spinal cord. Brain Res 1987;413:213-219.

16 Nabeshima T, Katoh A, Wada M, Kameyama T: Stress-induced changes in brain met-enkephalin, leu-enkephalin and dynorphin concentrations. Life Sci 1992;51:211-217.

17 McLaughlin JP, Marton-Popovici M, Chavkin C: Kappa opioid receptor antagonism and prodynorphin gene disruption block stress-induced behavioral responses. J Neurosci 2003;23:5674-5683.

18 Contet C, Gaveriaux-Ruff C, Matifas A, Caradec C, Champy MF, Kieffer BL: Dissociation of analgesic and hormonal responses to forced swim stress using opioid receptor knockout mice. Neuropsychopharmacology 2006;31:1733-1744.

19 Bruchas MR, Land BB, Aita M, Xu M, Barot SK, Li S, Chavkin C: Stress-induced p38 mitogen-activated protein kinase activation mediates kappa-opioid-dependent dysphoria. J Neurosci 2007;27:11614-11623.

20 Carlezon WA Jr, Beguin C, DiNieri JA, Baumann MH, Richards MR, Todtenkopf MS, Rothman RB, Ma Z, Lee DY, Cohen BM: Depressive-like effects of the kappa-opioid receptor agonist salvinorin $\mathrm{A}$ on behavior and neurochemistry in rats. J Pharmacol Exp Ther 2006;316:440-447.

21 Kuzmin AV, Semenova S, Gerrits MA, Zvartau EE, Van Ree JM: Kappa-opioid receptor agonist U50,488 h modulates cocaine and morphine self-administration in drug-naive rats and mice. Eur J Pharmacol 1997;321: 265-271.

22 Glick SD, Maisonneuve IM, Raucci J, Archer S: Kappa opioid inhibition of morphine and cocaine self-administration in rats. Brain Res 1995;681:147-152.

23 Land BB, Bruchas MR, Lemos JC, Xu M, Melief EJ, Chavkin C: The dysphoric component of stress is encoded by activation of the dynorphin kappa-opioid system. J Neurosci 2008;28:407-414.
24 Chavkin C: Stress-induced dysphoria is encoded by the dynorphin-kappa opioid system. Faseb J 2008;22:251-254.

-25 Xu M, Bruchas MR, Ippolito DL, Gendron L, Chavkin C: Sciatic nerve ligation-induced proliferation of spinal cord astrocytes is mediated by kappa opioid activation of p38 mitogen-activated protein kinase. J Neurosci 2007;27:2570-2581.

26 McLaughlin JP, Xu M, Mackie K, Chavkin C: Phosphorylation of a carboxyl-terminal serine within the kappa-opioid receptor produces desensitization and internalization. J Biol Chem 2003;278:34631-34640.

27 Svingos AL, Colago EE, Pickel VM: Cellular sites for dynorphin activation of kappa-opioid receptors in the rat nucleus accumbens shell. J Neurosci 1999;19:1804-1813.

28 Stiene-Martin A, Knapp PE, Martin K, Gurwell JA, Ryan S, Thornton SR, Smith FL, Hauser KF: Opioid system diversity in developing neurons, astroglia, and oligodendroglia in the subventricular zone and striatum: impact on gliogenesis in vivo. Glia 2001;36: 78-88.

29 Vaysse PJ, Zukin RS, Fields KL, Kessler JA: Characterization of opioid receptors in cultured neurons. J Neurochem 1990;55:624631.

30 Herraez-Baranda LA, Carretero J, GonzalezSarmiento R, Rodriguez RE: Kappa opioid receptor is expressed in the rat cerebellar cortex. Cell Tissue Res 2005;320:223-228.

-31 Martin JA, Kung HC, Mathews TJ, Hoyert DL, Strobino DM, Guyer B, Sutton SR: Annual summary of vital statistics: 2006. Pediatrics 2008;121:788-800.

- 32 Carbajal R, Lenclen R, Jugie M, Paupe A, Barton BA, Anand KJ: Morphine does not provide adequate analgesia for acute procedural pain among preterm neonates. Pediatrics 2005; 115:1494-1500

33 Orsini AJ, Leef KH, Costarino A, Dettorre MD, Stefano JL: Routine use of fentanyl infusions for pain and stress reduction in infants with respiratory distress syndrome. J Pediatr 1996;129:140-145.

34 Kreek MJ, Koob GF: Drug dependence: stress and dysregulation of brain reward pathways. Drug Alcohol Depend 1998;51:23-47.

- 35 Heikkila L, Rimon R, Terenius L: Dynorphin a and substance $p$ in the cerebrospinal fluid of schizophrenic patients. Psychiatry Res 1990;34:229-236.

36 Palkovits M: Stress-induced expression of co-localized neuropeptides in hypothalamic and amygdaloid neurons. Eur J Pharmacol 2000;405:161-166. 